\title{
Reflection and Transmission at the Boundary Surface of Thermoelastic Swelling Porous Media
}

\author{
Rajneesh Kumar ${ }^{1}$, Divya Taneja ${ }^{2 *}$ and Kuldeep Kumar ${ }^{2}$ \\ ${ }^{1}$ Department of Mathematics, Kurukshetra University, Kurukshetra, Haryana, India \\ ${ }^{2}$ Department of Mathematics, National Institute of Technology, Kurukshetra, Haryana, India
}

\begin{abstract}
The present investigation is concerned with the propagation of waves and their reflection and transmission from a plane interface between two different swelling porous thermo elastic half-spaces in welded contact. It is shown that there exist two dilatational waves, thermal wave and two transverse waves propagating with different velocities. The amplitude ratios of various reflected and transmitted waves are computed and presented graphically. It is found that amplitude ratios of reflected and transmitted waves are functions of angle of incidence, frequency and swelling porosity of the media. A particular case has also been deduced from the present investigation. The present investigation has immense application in geophysics and manufactured materials.
\end{abstract}

Keywords: Swelling porous media; Amplitude ratios; Longitudinal waves; Transversal waves

\section{Introduction}

The continuum theory of mixtures is extensively studied in literature. In this theory, a greater abstraction was achieved by assuming that the constituents of a mixture could be modeled as superimposed continua, so that each point in the mixture was simultaneously occupied by a material point of each constituent. Eringen [1] has developed a continuum theory of swelling porous elastic soils as a continuum theory of mixture consisting of three components an elastic solid, a viscous fluid and a gas. He used second law of thermodynamics to develop general and linear constitutive equations. Swelling porous medium (material) is a porous material that swells (shrinks) upon whetting (drying). Swelling porous elastic solid is a special case of continuous theory of mixture which consists of elastic solid, a viscous fluid \& a gas. Biot $[2,3]$ was the first who developed a linear theory for a fluid saturated porous elastic solid. He showed that there exist two kinds of compressional waves (one fast and other slow) along with a transverse wave in a fluid saturated porous medium. Biot theory was based on the assumption of compressible constituents and till recently, some of his results have been taken as standard references and the basis for subsequent analysis in acoustic, geophysics and other such fields. Based on the Fillunger model [4], (which is further based on the concept of volume fractions combined with surface porosity coefficients), Bowen [5], Boer and Ehlers [6,7] and Ehlers [8] developed and used another interesting theory in which all the constituents of a porous medium are assumed of soil; solid constituents are incompressible and liquid constituents which are generally water or oils are also incompressible.

Angel and Achenbach [9] studied the reflection and transmission of elastic waves by a periodic array of crack. Tomar and Kumar [10] obtain the amplitude ratios of reflection and transmission of longitudinal displacement wave at a liquid micropolar solid interface. Sinha and Elasibai [11] studied the reflection and refraction of thermoelastic waves at an interface of two semi-infinite media with two relaxation times. Singh and Kumar [12,13] studied the reflection and refraction of waves in micropolar elastic solid. Tomar et al. [14], studied the reflection and refraction of SH waves at corrugated interface between transversely isotropic and visco-elastic solid half space. Kumar and Sarthi [15] studied the reflection and refraction of thermo elastic plane waves at an interface between two thermo elastic media without energy dissipation. Chattopadhyay et al. [16], studied the reflection and refraction of plane
quasi-P waves at a corrugated interface between distinct triclinic elastic half-space.

In the present problem, we have studied the reflection and transmission of waves at an interface of two swelling porous thermo elastic media. Amplitude ratios have been obtained and the results are compared with and without swelling porous thermoelastic (EL) medium. The present problem has immense application in the inspection of geological materials, rocks and manufactured materials.

\section{Basic Equation}

Following Eringen [1], the field equations in linear theory of swelling porous elastic soils are

$$
\begin{aligned}
& \mu u_{i, j j}^{s}+(\lambda+\mu) u_{j, j i}^{s}-\sigma^{f} u_{j, j i}^{f}+\xi^{f f}\left(\dot{u}_{i}^{f}-\dot{u}_{i}^{s}\right)+\left(\gamma^{f}-\alpha_{0}\right) \nabla T+f_{i}^{s}=\rho_{0}^{s} \ddot{u}_{i}^{s},(1) \\
& \mu_{v} \dot{u}_{i, j j}^{f}+\left(\lambda_{v}+\mu_{v}\right) \dot{u}_{j, j i}^{f}-\sigma^{f} u_{j, j i}^{s}-\sigma^{f f} u_{j, j i}^{f}-\xi^{f f}\left(\dot{u}_{i}^{f}-\dot{u}_{i}^{s}\right)-\left(\alpha^{f}+\gamma^{f}\right) \nabla T+f_{i}^{f}=\rho_{0}^{f} \dot{u}_{i}^{f}, \\
& \alpha_{1} \dot{T}-\varpi \ddot{T}+\left(\frac{\zeta^{f}}{T_{0}}+\alpha^{f}\right) \nabla \cdot \dot{u}^{f}+\left(\alpha_{0}-\frac{\zeta^{f}}{T_{0}}\right) \nabla \cdot \dot{u}^{s}-\frac{K^{*}}{T_{0}} \nabla^{2} T=0 \\
& t_{i j}^{s}=\left(-\sigma^{f} u_{r, r}^{f}+\lambda u_{r, r}^{s}\right) \delta_{i j}+\mu\left(u_{i, j}^{s}+u_{j, i}^{s}\right) \\
& t_{i j}^{f}=\left(-\sigma^{f} u_{r, r}^{s}-\sigma^{f f} u_{r, r}^{f}+\lambda_{v} \dot{u}_{r, r}^{f}\right) \delta_{i j}+\mu_{v}\left(\dot{u}_{i, j}^{f}+\dot{u}_{j, i}^{f}\right)
\end{aligned}
$$$$
i, j=1,2,3
$$

where, the superscripts $s$ and $f$ denote respectively, the elastic solid and the fluid; $u_{i}^{s}$ and $u_{i}^{f}$ are the displacement components of solid and fluid respectively. The functions are $\left(f_{i}^{s}, f_{i}^{f}\right)$ the body forces, $\rho_{0}^{s}, \rho_{0}^{f}$ are the densities of each constituent, $\lambda, \mu, \lambda, \mu_{v}, \sigma^{f}, \sigma^{f f}, \xi^{f f}$ are constitutive constants, $\alpha_{1}, \varpi, \zeta^{f}, \alpha^{f}, \alpha_{0}$ are material constant, $\mathrm{T}$ is temperature and $K^{*}$ is thermal conductivity. Subscripts preceded by a

*Corresponding author: Divya Taneja, Department of Mathematics, National Institute of Technology, Kurukshetra, Haryana, 136119, India; Email: divyataneja82@yahoo.com

Received February 22, 2012; Accepted March 29, 2012; Published March 31, 2012

Citation: Kumar R, Taneja D, Kumar K (2012) Reflection and Transmission at the Boundary Surface of Thermoelastic Swelling Porous Media. J Appl Mech Eng 1:104. doi:10.4172/2168-9873.1000104

Copyright: @ 2012 Kumar R, et al. This is an open-access article distributed unde the terms of the Creative Commons Attribution License, which permits unrestricted use, distribution, and reproduction in any medium, provided the original author and source are credited. 
comma denote partial differentiation with respect to the corresponding Cartesian coordinate, and a superposed dot denotes time differentiation, $t_{i j}^{s}, t_{i j}^{f}$ are the partial stress tensors.

\section{Formulation of the Problem and Solution}

We consider two homogenous, isotropic, swelling porous thermoelastic half space medium $\mathrm{M}_{1}\left(0<x_{3}<\infty\right)$ and medium $\bar{M}\left(-\infty<x_{3}<0\right)$. The media are in contact with each other at the plane surface, which we designate as the plane $x_{3}=0$ of the rectangular Cartesian coordinate system $O_{1} x_{2} x_{3}$ with an origin on the surface $x_{3}$ $=0$ and $x_{3}$-axis pointing vertically into the medium. The complete geometry of the problem is shown in Figure 1. We write all the variables without a hat in the region $x_{3}>0$ (medium $\mathrm{M}_{1}$ ) and attach a hat to denote the variables in the region $x_{3}<0$ (medium $\bar{M}$ ). For two dimensional problems, we assume the displacement vector

$$
\vec{u}^{i}=\left(u_{1}^{i}, 0, u_{3}^{i}\right) \quad \mathrm{i}=\mathrm{s}, \mathrm{f}
$$

We define the non-dimensional quantities as

$$
\begin{aligned}
& x_{1}^{\prime}=\frac{\omega^{*}}{c_{1}} x_{1}, x_{3}^{\prime}=\frac{\omega^{*}}{c_{1}} x_{3}, u_{1}^{i^{\prime}}=\frac{\rho_{0}^{s} \omega^{*} c_{1}}{\alpha_{0} T_{0}} u_{1}^{i}, u_{3}^{i^{\prime}}=\frac{\rho_{0}^{s} \omega^{*} c_{1}}{\alpha_{0} T_{0}} u_{3}^{i}, t_{i j}^{\prime i}=\frac{t_{i j}^{i}}{\alpha_{0} T_{0}}, t^{\prime}=\omega^{*} t, \\
& h^{\prime}=\frac{c_{1} h}{\omega^{*}}, \omega^{*}=\frac{T_{0} \alpha_{1} c_{1}^{2}}{K^{*}}, c_{1}^{2}=\frac{\lambda+2 \mu}{\rho_{0}^{s}}, T^{\prime}=\frac{T}{T_{0}}, \bar{u}_{1}^{i^{\prime}}=\frac{\bar{\rho}_{0}^{s} \bar{\omega}^{*} \bar{c}_{1}}{\bar{\alpha}_{0} \bar{T}_{0}} \bar{u}_{1}^{i}, \bar{u}_{3}^{i^{\prime}}=\frac{\bar{\rho}_{0}^{s} \bar{\omega}^{*} \bar{c}_{1}}{\bar{\alpha}_{0} \bar{T}_{0}} \bar{u}_{3}^{i}, \\
& \bar{t}_{i j}^{\prime i}=\frac{\bar{t}_{i j}^{i}}{\bar{\alpha}_{0} \bar{T}_{0}}, \bar{\omega}^{*}=\frac{\bar{T}_{0} \bar{\alpha}_{1} \bar{c}_{1}^{2}}{\bar{K}^{*}}, \bar{c}_{1}^{2}=\frac{\bar{\lambda}+2 \bar{\mu}}{\bar{\rho}_{0}^{s}}, \bar{T}^{\prime}=\frac{\bar{T}}{\bar{T}_{0}},
\end{aligned}
$$

The displacements components $u_{1}^{s}, u_{3}^{s}, u_{1}^{f}, u_{3}^{f}$ are related by the potential functions

$$
u_{1}^{i}=\frac{\partial \phi^{i}}{\partial x_{1}}-\frac{\partial \psi^{i}}{\partial x_{3}}, \quad u_{3}^{i}=\frac{\partial \phi^{i}}{\partial x_{3}}+\frac{\partial \psi^{i}}{\partial x_{1}}
$$

Equations (1)-(3) with the aid of (6) - (8) in absence of body forces and heat sources take the form,

$$
\begin{aligned}
& \left.\Delta_{2}-a_{2} \frac{\partial}{\partial t}-\frac{\partial^{2}}{\partial t^{2}}\right] \phi^{s}\left[-a_{1} \Delta_{2}+a_{2} \frac{\partial}{\partial t}\right] \phi^{f}-a_{3} T=0, \\
& {\left[-\delta_{1}^{2} \Delta_{2}+a_{2} \frac{\partial}{\partial t}+\frac{\partial^{2}}{\partial t^{2}}\right] \psi^{s}-a_{2} \frac{\partial}{\partial t} \psi^{f}=0,} \\
& {\left[\frac{\partial}{\partial t} \Delta_{2}-h_{2} \Delta_{2}-h_{3} \frac{\partial}{\partial t}-h_{s} \frac{\partial^{2}}{\partial t^{2}}\right] \phi^{f}\left[-h_{1} \Delta_{2}+h_{3} \frac{\partial}{\partial t}\right] \phi^{s}-h_{4} T=0,} \\
& {\left[-\delta_{2}^{2} \Delta_{2} \frac{\partial}{\partial t}+h_{3} \frac{\partial}{\partial t}+h_{s} \frac{\partial^{2}}{\partial t^{2}}\right] \psi^{f}-h_{3} \frac{\partial}{\partial t} \psi^{s}=0,} \\
& \left(1+b_{1} \frac{\partial}{\partial t}\right) \frac{\partial T}{\partial t}+b_{2}\left(\frac{\partial^{2} \dot{\phi}^{f}}{\partial x_{1}^{2}}+\frac{\partial^{2} \dot{\phi}^{f}}{\partial x_{3}^{2}}\right)+b_{3}\left(\frac{\partial^{2} \phi^{s}}{\partial x_{1}^{2}}+\frac{\partial^{2} \phi^{s}}{\partial x_{3}^{2}}\right)=\left(\frac{\partial^{2}}{\partial x_{1}^{2}}+\frac{\partial^{2}}{\partial x_{3}^{2}}\right) T, \\
& t_{33}^{s}=\frac{\lambda}{\rho_{0}^{s} c_{1}^{2}}\left(\frac{\partial^{2} \phi^{s}}{\partial x_{1}^{2}}+\frac{\partial^{2} \phi^{s}}{\partial x_{3}^{2}}\right)-\frac{\sigma^{f}}{\rho_{0}^{s} c_{1}^{2}}\left(\frac{\partial^{2} \phi^{f}}{\partial x_{1}^{2}}+\frac{\partial^{2} \phi^{f}}{\partial x_{3}^{2}}\right)+2 \frac{\mu}{\rho_{0}^{s} c_{1}^{2}}\left(\frac{\partial^{2} \phi^{s}}{\partial x_{3}^{2}}+\frac{\partial^{2} \psi^{s}}{\partial x_{1} \partial x_{3}}\right), \\
& t_{33}^{f}=-\frac{\sigma^{f}}{\rho_{0}^{s} c_{1}^{2}}\left(\frac{\partial^{2} \phi^{s}}{\partial x_{1}^{2}}+\frac{\partial^{2} \phi^{s}}{\partial x_{3}^{2}}\right)+\left(-\frac{\sigma^{f}}{\rho_{0}^{s} c_{1}^{2}}+\frac{\lambda_{v} \omega^{*}}{\rho_{0}^{s} c_{1}^{2}} \frac{\partial}{\partial t}\right)\left(\frac{\partial^{2} \phi^{f}}{\partial x_{1}^{2}}+\frac{\partial^{2} \phi^{f}}{\partial x_{3}^{2}}\right)+\frac{2 \mu_{s} \omega^{*}}{\rho_{0}^{s} c_{1}^{2}}\left(\frac{\partial^{2} \dot{\phi}^{f}}{\partial x_{3}^{2}}+\frac{\partial^{2} \dot{\psi}^{f}}{\partial x_{1} \partial x_{3}}\right), \\
& t_{31}^{s}=\frac{\mu}{\rho_{0}^{s} c_{1}^{2}}\left(2 \frac{\partial^{2} \phi^{s}}{\partial x_{1} \partial x_{3}}+\frac{\partial^{2} \psi^{s}}{\partial x_{1}^{2}}-\frac{\partial^{2} \psi^{s}}{\partial x_{3}^{2}}\right) \\
& t_{31}^{f}=\frac{\mu_{v} \omega^{s}}{\rho_{0}^{s} c_{1}^{2}} \frac{\partial}{\partial t}\left(2 \frac{\partial^{2} \phi^{f}}{\partial x_{1} \partial x_{3}}+\frac{\partial^{2} \psi^{f}}{\partial x_{1}^{2}}-\frac{\partial^{2} \psi^{f}}{\partial x_{3}^{2}}\right),
\end{aligned}
$$

Where,

$$
\begin{aligned}
& a_{1}=\frac{\sigma^{f}}{\lambda+2 \mu}, \quad a_{2}=\frac{\xi^{f f}}{\rho_{0}^{s} \omega^{*}}, \quad a_{3}=\left(1-\tau_{0}\right), \tau_{0}=\frac{\gamma^{f}}{\alpha_{0}}, \\
& h_{1}=\frac{\sigma^{f}}{\left(\lambda_{v}+2 \mu_{v}\right) \omega^{*}}, \quad h_{2}=\frac{\sigma^{f f}}{\left(\lambda_{v}+2 \mu_{v}\right) \omega^{*}}
\end{aligned}
$$

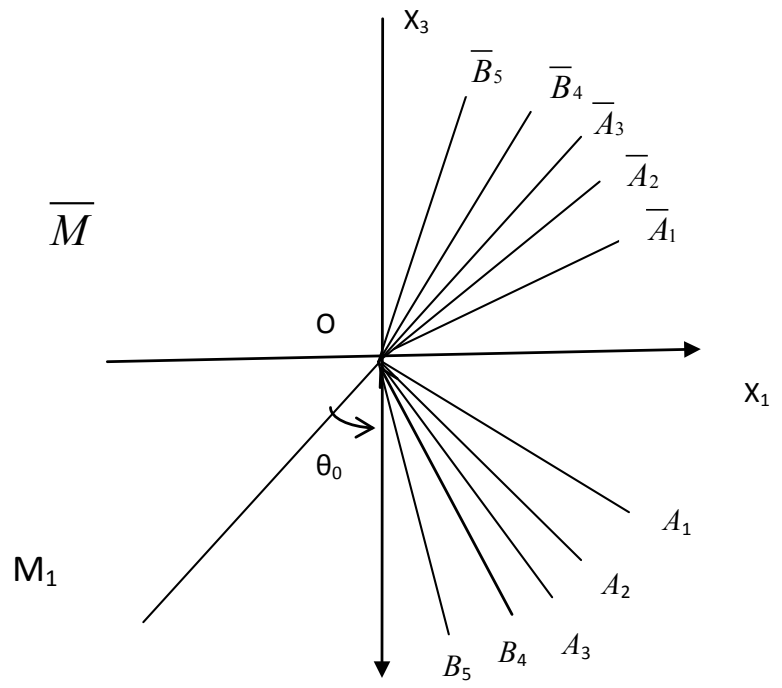

Figure 1: Geometry of the problem

$$
\begin{aligned}
& h_{3}=\frac{\xi^{f f} c_{1}^{2}}{\omega^{* 2}\left(\lambda_{v}+2 \mu_{v}\right)}, h_{4}=\frac{\alpha^{f} c_{1}^{2} \rho_{0}^{s}\left(1+\tau_{1}\right)}{\omega^{*} \alpha_{0}\left(\lambda_{v}+2 \mu_{v}\right)}, h_{5}=\frac{c_{1}^{2} \rho_{0}^{f}}{\omega^{*}\left(\lambda_{v}+2 \mu_{v}\right)}, \\
& b_{1}=-\tau_{2} \omega^{*}, b_{2}=\frac{\beta_{2} \alpha_{0}}{\rho_{0}^{s} \alpha_{1} c_{1}^{2} T_{0}}, b_{3}=\frac{\beta_{1} \alpha_{0}}{\rho_{0}^{s} \alpha_{1} c_{1}^{2} T_{0}} \\
& \tau_{1}=\frac{\gamma^{f}}{\alpha^{f}}, \tau_{2}=\frac{\sigma}{\alpha_{1}}, \beta_{1}=\alpha_{0} T_{0}-\zeta^{f}, \beta_{2}=\alpha^{f} T_{0}+\zeta^{f}, \\
& \delta_{1}^{2}=\frac{\mu}{\lambda+2 \mu}, \delta_{2}^{2}=\frac{\mu_{v}}{\left(\lambda_{v}+2 \mu_{v}\right)}
\end{aligned}
$$

\section{Reflection and Transmission}

We consider a longitudinal wave in solid (PS)/longitudinal wave in fluid (PF)/thermal wave (T)/transverse wave in solid (SVS)/ transverse wave in fluid(SVF) propagating through the medium $M_{1}$ which is designated as the region $x_{3}>0$ and incident at the plane $x_{3}=0$, with its direction of propagation with angle $\theta_{0}$ normal to the surface. Corresponding to each incident wave, we get reflected PS, PF, T, SVS, SVF waves in medium $M_{1}$ and transmitted PS, PF, T, SVS, SVF waves in medium $\bar{M}$ as shown in Figure 1 .

\section{Boundary conditions}

The boundary conditions at $\mathrm{x}_{3}=0$ are:

(i) $t_{33}^{s}=\bar{t}_{33}^{s}$ (ii) $t_{33}^{f}=\bar{t}_{33}^{f}$ (iii) $t_{31}^{s}=\bar{t}_{31}^{s}$ (iv) $t_{31}^{f}=\bar{t}_{31}^{f}$

(v) $\dot{u}_{3}^{s}-\dot{\bar{u}}_{3}^{s}=0 \quad(v i) \dot{u}_{3}^{f}-\dot{\bar{u}}_{3}^{f}=0 \quad(v i i) \dot{u}_{1}^{s}-\dot{\bar{u}}_{1}^{s}=0 \quad$ (viii) $\dot{u}_{1}^{f}-\dot{\bar{u}}_{1}^{f}=0$

(ix) $K^{*} \frac{\partial T}{\partial x_{3}}=\bar{K}^{*} \frac{\partial \bar{T}}{\partial x_{3}}$

We assume the values of $\phi^{s}, \phi^{f}, T, \psi^{s}, \psi^{f}$ for medium $\mathrm{M}_{1}$ and $\bar{\phi}^{s}, \bar{\phi}^{f}, \bar{T}, \bar{\psi}^{s}, \bar{\psi}^{f}$ for medium $\bar{M}$ satisfying the boundary conditions as

$$
\begin{aligned}
& \left\{\phi^{s}, \phi^{f}, T\right\}=\sum_{i=1}^{3}\left\{1, \eta_{i}, \xi_{i}\right\}\left[A_{0 i} e^{i\left\{k_{i}\left\{x_{1} \sin \theta_{\theta_{i}}-x_{3} \cos \theta_{0 i}\right\}-\omega_{i} t\right\}}+P_{i}\right] \\
& \left\{\psi^{s}, \psi^{f}\right\}=\sum_{j=4}^{5}\left\{1, \gamma_{j}\right\}\left[B_{0 j} e^{i\left\{k_{j}\left\{x_{i} \sin \theta_{\theta_{j}}-x_{3} \cos \theta_{\theta_{j}}\right\}-\omega_{j} t\right\}}+P_{j}\right] \\
& \left\{\bar{\phi}^{s}, \bar{\phi}^{f}, \bar{T}\right\}=\sum_{i=1}^{3}\left\{1, \bar{\eta}_{i}, \bar{\xi}_{i}\right\}\left[\bar{A}_{i} e^{i\left\{\bar{k}_{i}\left\{x_{1} \sin \bar{\theta}_{i}-x_{3} \cos \bar{\theta}_{i}\right\}-\bar{\omega}_{i} t\right\}}\right]
\end{aligned}
$$




$$
\left\{\bar{\psi}^{s}, \bar{\psi}^{f}\right\}=\sum_{j=4}^{5}\left\{1, \bar{\gamma}_{j}\right\}\left[\bar{B}_{j} e^{i\left\{\bar{k}_{j}\left\{x_{1} \sin \bar{\theta}_{j}-x_{3} \cos \bar{\theta}_{j}\right\}-\bar{\omega}_{j} t\right\}}\right]
$$

Where,

$$
\begin{aligned}
& P_{i}=A_{i} e^{i\left\{k_{i}\left\{x_{1} \sin \theta_{i}+x_{3} \cos \theta_{i}\right\}-\omega_{i} t\right\}}, P_{j}=B_{j} e^{i\left\{k_{j}\left\{x_{1} \sin \theta_{j}+x_{3} \cos \theta_{j}\right\}-\omega_{j} t\right\}}, \\
& \eta_{i}=\frac{\left(\omega h_{1}-h_{3} i V_{i}^{2}\right)\left(i V_{i}^{2}+\omega V_{i}^{2} b_{1}-\omega\right)-i b_{3} \omega h_{4} V_{i}^{2}}{\left[\omega^{2}\left(i+h_{2}\right)+\omega V_{i}^{2}\left(i h_{3}+h_{5}\right)\right]\left[-V_{i}^{2}\left(i+\omega b_{1}\right)+\omega\right]+i b_{2} h_{4} V_{i}^{2} \omega} \\
& \xi_{i}=\frac{\left(\omega^{2} h_{1}-h_{3} i \omega V_{i}^{2}\right) i b_{2}-i b_{3}\left[\omega^{3}\left(i+h_{2}\right)+V_{i}^{2} \omega^{2}\left(i h_{3}+h_{5}\right)\right]}{\left[\omega\left(i+h_{2}\right)+V_{i}^{2}\left(i h_{3}+h_{5}\right)\right]\left[-V_{i}^{2}\left(i+\omega b_{1}\right)+\omega\right]+i b_{2} h_{4} V_{i}^{2}}, \\
& \gamma_{j}=\frac{\frac{h_{3} i V_{j}^{2}}{\omega}}{\delta_{2}^{2} i \omega+h_{5} V_{j}^{2}+\frac{h_{3} i V_{j}^{2}}{\omega}} \\
& \bar{\eta}_{i}=\frac{\left(\omega \bar{h}_{1}-\bar{h}_{3} i \bar{V}_{i}^{2}\right)\left(i \bar{V}_{i}^{2}+\omega \bar{V}_{i}^{2} \bar{b}_{1}-\omega\right)-i \bar{b}_{3} \omega \bar{h}_{4} \bar{V}_{i}^{2}}{\left[\omega^{2}\left(i+\bar{h}_{2}\right)+\omega \bar{V}_{i}^{2}\left(i \bar{h}_{3}+\bar{h}_{5}\right)\right]\left[-\bar{V}_{i}^{2}\left(i+\omega \bar{b}_{1}\right)+\omega\right]+i \bar{b}_{2} \bar{h}_{4} \bar{V}_{i}^{2} \omega} \\
& \bar{\xi}_{i}=\frac{\left(\omega^{2} \bar{h}_{1}-\bar{h}_{3} i \omega \bar{V}_{i}^{2}\right) i \bar{b}_{2}-i \bar{b}_{3}\left[\omega^{3}\left(i+\bar{h}_{2}\right)+\bar{V}_{i}^{2} \omega^{2}\left(i \bar{h}_{3}+\bar{h}_{5}\right)\right]}{\left[\omega\left(i+\bar{h}_{2}\right)+\bar{V}_{i}^{2}\left(i \bar{h}_{3}+\bar{h}_{5}\right)\right]\left[-\bar{V}_{i}^{2}\left(i+\omega \bar{b}_{1}\right)+\omega\right]+i \bar{b}_{2} \bar{h}_{4} \bar{V}_{i}^{2}}, \\
& \bar{\gamma}_{j}=\frac{\bar{h}_{3} i \bar{V}_{j}^{2}}{\omega} \\
& (i=1,2,3 \& j=4,5)
\end{aligned}
$$

$A_{0 i}$ are the amplitudes of the incident PS wave, PF wave, T wave and $B_{0 j}$ are the amplitudes of the incident SVS wave, SVF wave respectively. $A_{i}$ are the amplitudes of the reflected PS wave (PSR), PF wave (PFR), T wave (TR) and $B_{j}$ are the amplitudes of the reflected SVS wave (SVSR) and SVF wave (SVFR) , $\bar{A}_{i}$ are the amplitudes of the transmitted PS wave (PST), PF wave (PFT), T wave (TT), $\bar{B}_{j}$ are the amplitudes of transmitted SVS wave (SVST), SVF wave (SVFT) respectively.

In order to satisfy the boundary conditions, the extension of the Snell's law will be

$$
\begin{aligned}
& \frac{\sin \theta_{0}}{V_{0}}=\frac{\sin \theta_{1}}{V_{1}}=\frac{\sin \theta_{2}}{V_{2}}=\frac{\sin \theta_{3}}{V_{3}}=\frac{\sin \theta_{4}}{V_{4}}= \\
& \frac{\sin \theta_{5}}{V_{5}}=\frac{\sin \bar{\theta}_{1}}{\bar{V}_{1}}=\frac{\sin \bar{\theta}_{2}}{\bar{V}_{2}}=\frac{\sin \bar{\theta}_{3}}{\bar{V}_{3}}=\frac{\sin \bar{\theta}_{4}}{\bar{V}_{4}}=\frac{\sin \bar{\theta}_{5}}{\bar{V}_{5}}
\end{aligned}
$$

Where

$$
k_{1} V_{1}=k_{2} V_{2}=k_{3} V_{3}=k_{4} V_{4}=k_{5} V_{5}=\bar{k}_{1} \bar{V}_{1}=\bar{k}_{2} \bar{V}_{2}=\bar{k}_{3} \bar{V}_{3}=\bar{k}_{4} \bar{V}_{4}=\bar{k}_{5} \bar{V}_{5}=\omega
$$

at $x_{3}=0$

Making use of potentials given by Equation (19) - (22) in the boundary conditions, we obtain a system of ten non-homogeneous equations which can be written as

$$
\sum_{i, j=1}^{10} a_{i j} Z_{j}=Y_{i}
$$

Where,

$$
\begin{aligned}
& a_{1 i}=\left(\frac{\sigma^{f}}{\rho_{0}^{s} c_{1}^{2}} \eta_{i}-\frac{\lambda}{\rho_{0}^{s} c_{1}^{2}}-\frac{2 \mu \cos ^{2} \theta_{i}}{\rho_{0}^{s} c_{1}^{2}}\right) \frac{V_{1}^{2}}{V_{i}^{2}}, a_{1 j}=\left(\frac{2 V_{1}^{2} \mu \cos \theta_{j} \sin \theta_{j}}{V_{j}^{2} \rho_{0}^{s} c_{1}^{2}}\right), \\
& a_{1 k}=-\left(\frac{\bar{\sigma}^{f}}{\bar{\rho}_{0}^{s} \bar{c}_{1}^{2}} \bar{\eta}_{i}-\frac{\bar{\lambda}}{\bar{\rho}_{0}^{s} \bar{c}_{1}^{2}}-\frac{2 \bar{\mu} \cos ^{2} \bar{\theta}_{i}^{2}}{\bar{\rho}_{0}^{s} \bar{c}_{1}^{2}}\right) \frac{V_{1}^{2}}{\bar{V}_{i}^{2}}, \\
& a_{11=}\left(\frac{-2 V_{1}^{2} \bar{\mu} \cos \bar{\theta}_{j} \sin \bar{\theta}_{j}}{\bar{V}_{j}^{2} \bar{\rho}_{0}^{s} \bar{c}_{1}^{2}}\right), a_{2 i}=\left(\frac{\sigma^{f}}{\rho_{0}^{s} c_{1}^{2}}+\frac{\sigma^{f f} \eta_{i}}{\rho_{0}^{s} c_{1}^{2}}+\frac{i \omega \omega^{*} \eta_{i}}{\rho_{0}^{s} c_{1}^{2}}\left(\lambda_{v}+2 \mu_{v} \cos ^{2} \theta_{i}\right)\right) \frac{V_{1}^{2}}{V_{i}^{2}}, \\
& a_{2,=}=\left(\frac{-2 i \omega \mu_{v} \omega^{*} \gamma_{j} \cos \theta_{j} \sin \theta_{j}}{\rho_{0}^{s} c_{1}^{2}}\right) \frac{V_{1}^{2}}{V_{j}^{2}}, a_{2 k}=-\left(\frac{\bar{\sigma}^{f}}{\bar{\rho}_{0}^{s} \bar{c}_{1}^{2}}+\frac{\bar{\sigma}^{f f} \bar{\eta}_{i}}{\bar{\rho}_{0}^{s} \bar{c}_{1}^{2}}+\frac{i \omega \bar{\omega}^{s} \bar{\eta}_{i}}{\bar{\rho}_{0}^{s} \bar{c}_{1}^{2}}\left(\bar{\lambda}_{v}+2 \bar{\mu}_{v} \cos ^{2} \bar{\theta}_{i}\right)\right) \frac{V_{1}^{2}}{\bar{V}_{i}^{2}},
\end{aligned}
$$

$a_{21}=\left(\frac{2 i \omega \bar{\mu}_{i} \omega^{*} \bar{\gamma}_{j} \cos \bar{\theta}_{j} \sin \bar{\theta}_{j}}{\bar{\rho}_{0}^{s} \bar{c}_{1}^{2}}\right) \frac{V_{1}^{2}}{\bar{V}_{j}^{2}}, a_{3 i}=\frac{V_{1}^{2} 2 \mu \cos \theta_{i} \sin \theta_{i}}{V_{i}^{2} \rho_{0}^{s} c_{1}^{2}}, a_{3 j}=\frac{V_{1}^{2} \mu}{V_{j}^{2} \rho_{0}^{s} c_{1}^{2}}\left(-\sin ^{2} \theta_{j}+\cos ^{2} \theta_{j}\right)$,

$a_{3 k}=\frac{-2 V_{1}^{2} \omega \bar{\mu} \cos \bar{\theta}_{i} \sin \bar{\theta}_{i}}{\bar{V}_{i}^{2} \bar{\rho}_{0}^{s} \bar{c}_{1}^{2}}, a_{3 l}=\frac{V_{1}^{2} \bar{\mu}}{\bar{V}_{j}^{2} \bar{\rho}_{0}^{s} \bar{c}_{1}^{2}}\left(\sin ^{2} \bar{\theta}_{j}-\cos ^{2} \bar{\theta}_{j}\right)$,

$a_{4 i}=\left(\frac{-2 i \omega \mu_{v} \omega^{*} \eta_{i} \cos \theta_{i} \sin \theta_{i}}{\rho_{0}^{s} c_{1}^{2}}\right) \frac{V_{1}^{2}}{V_{i}^{2}}$,

$a_{4 j}=\frac{i \omega \mu_{v} \omega^{*} \gamma_{j}}{\rho_{0}^{s} c_{1}^{2}}\left(\sin ^{2} \theta_{j}-\cos ^{2} \theta_{j}\right) \frac{V_{j}^{2}}{V_{1}^{2}}, a_{4 k}=\left(\frac{2 i \omega \overline{\mu_{v}} \bar{\omega}^{*} \bar{\eta}_{i} \cos \bar{\theta}_{i} \sin \bar{\theta}_{i}}{\bar{\rho}_{0}^{s} \bar{c}_{1}^{2}}\right) \frac{V_{1}^{2}}{\bar{V}_{i}^{2}}$,

$a_{4 i}=\frac{i \omega \bar{\mu}_{v} \bar{\omega}^{*} \bar{\gamma}_{j}}{\bar{\rho}_{0}^{s} \bar{c}_{1}^{2}}\left(\cos ^{2} \bar{\theta}_{j}-\sin ^{2} \bar{\theta}_{j}\right) \frac{V_{1}^{2}}{\bar{V}_{j}^{2}}, a_{5 i}=-\frac{V_{1}}{V_{i}} i \cos \theta_{i}$,

$a_{5 j}=i \frac{V_{1}}{V_{j}} \sin \theta_{j}, a_{5 k}=i \frac{V_{1}}{\bar{V}_{i}} \cos \bar{\theta}_{i}, a_{5 l}=-i \frac{V_{1}}{\bar{V}_{j}} \sin \bar{\theta}_{j}$

$a_{6 i}=-\frac{V_{1}}{V_{i}} i \eta_{i} \cos \theta_{i}, a_{6 j}=\frac{V_{i}}{V_{j}} i \gamma_{j} \sin \theta_{j}, a_{6 k}=\frac{V_{1}}{\bar{V}_{i}} i \bar{\eta}_{i} \cos \bar{\theta}_{i}$,

$a_{6 l}=-\frac{V_{1}}{\bar{V}_{j}} i \bar{\gamma}_{j} \sin \bar{\theta}_{j}, a_{7 i}=i \frac{V_{1}}{V_{i}} \sin \theta_{i}$

$a_{7 j}=i \frac{V_{i}}{V_{j}} \cos \theta_{j}, a_{7 k}=-i \frac{V_{1}}{\bar{V}_{i}} \sin \bar{\theta}_{i}, a_{7 l}=-i \frac{V_{1}}{\bar{V}_{j}} \cos \bar{\theta}_{j}$,

$a_{8 i}=i \frac{V_{1}}{V_{i}} \eta_{i} \sin \theta_{i}, a_{8 j}=i \frac{V_{1}}{V_{j}} \gamma_{j} \cos \theta_{j}, a_{8 k}=-i \frac{V_{1}}{\bar{V}_{i}} \bar{\eta}_{i} \sin \bar{\theta}_{i}$,

$a_{8 l}=-i \frac{V_{1}}{\bar{V}_{j}} \bar{\eta}_{j} \cos \bar{\theta}_{j}, a_{9 i}=-i \frac{V_{1}}{V_{i}} \xi_{i} \cos \theta_{i}, a_{9 j}=0$,

$a_{9 k}=-i \frac{V_{1} \bar{K}^{*}}{\bar{V}_{i} K^{*}} \bar{\xi}_{i} \cos \bar{\theta}_{i}, a_{9 l}=0, a_{10 i}=\xi_{i}, a_{10 j}=0, a_{10 k}=-\bar{\xi}_{i}, a_{10 l}=0$

$(i=1,23 ; j=4,5 ; k=6,7,8 ; 1=9,10)$

$$
\begin{aligned}
& Z_{1}=\frac{A_{1}}{A^{*}}, Z_{2}=\frac{A_{2}}{A^{*}}, Z_{3}=\frac{A_{3}}{A^{*}}, Z_{4}=\frac{B_{4}}{A^{*}}, Z_{5}=\frac{B_{5}}{A^{*}}, \\
& Z_{6}=\frac{\bar{A}_{1}}{A^{*}}, Z_{7}=\frac{\bar{A}_{2}}{A^{*}}, Z_{8}=\frac{\bar{A}_{3}}{A^{*}}, Z_{9}=\frac{\bar{B}_{4}}{A^{*}}, Z_{10}=\frac{\bar{B}_{5}}{A^{*}},
\end{aligned}
$$

(i)For incident PS -wave:

$$
\begin{aligned}
& A^{*}=A_{01}, A_{02}=A_{02}=B_{04}=B_{05}=0 \\
& Y_{1}=-a_{11}, Y_{2}=-a_{21}, Y_{3}=a_{31}, Y_{4}=a_{41}, Y_{5}=a_{51,}, \\
& Y_{6}=a_{61}, Y_{7}=-a_{71}, Y_{8}=-a_{81}, Y_{9}=a_{91}, Y_{10}=-a_{101}
\end{aligned}
$$

(ii) For incident PF-wave:

$$
\begin{aligned}
& A^{*}=A_{02}, A_{01}=A_{03}=B_{04}=B_{05}=0 \\
& Y_{1}=-a_{12}, Y_{2}=-a_{22}, Y_{3}=a_{32}, Y_{4}=a_{42}, Y_{5}=a_{52,} \\
& Y_{6}=a_{62}, Y_{7}=-a_{72}, Y_{8}=-a_{82}, Y_{9}=a_{92}, Y_{10}=-a_{102}
\end{aligned}
$$

(iii) For incident T-wave:

$$
\begin{aligned}
& A^{*}=A_{03}, A_{01}=A_{02}=B_{04}=B_{05}=0 \\
& Y_{1}=-a_{13}, Y_{2}=-a_{23}, Y_{3}=a_{33}, Y_{4}=a_{43}, Y_{5}=a_{53}, \\
& Y_{6}=a_{63}, Y_{7}=-a_{73}, Y_{8}=-a_{83}, Y_{9}=a_{93}, Y_{10}=-a_{103}
\end{aligned}
$$

(iii) For incident SVS -wave: 
A
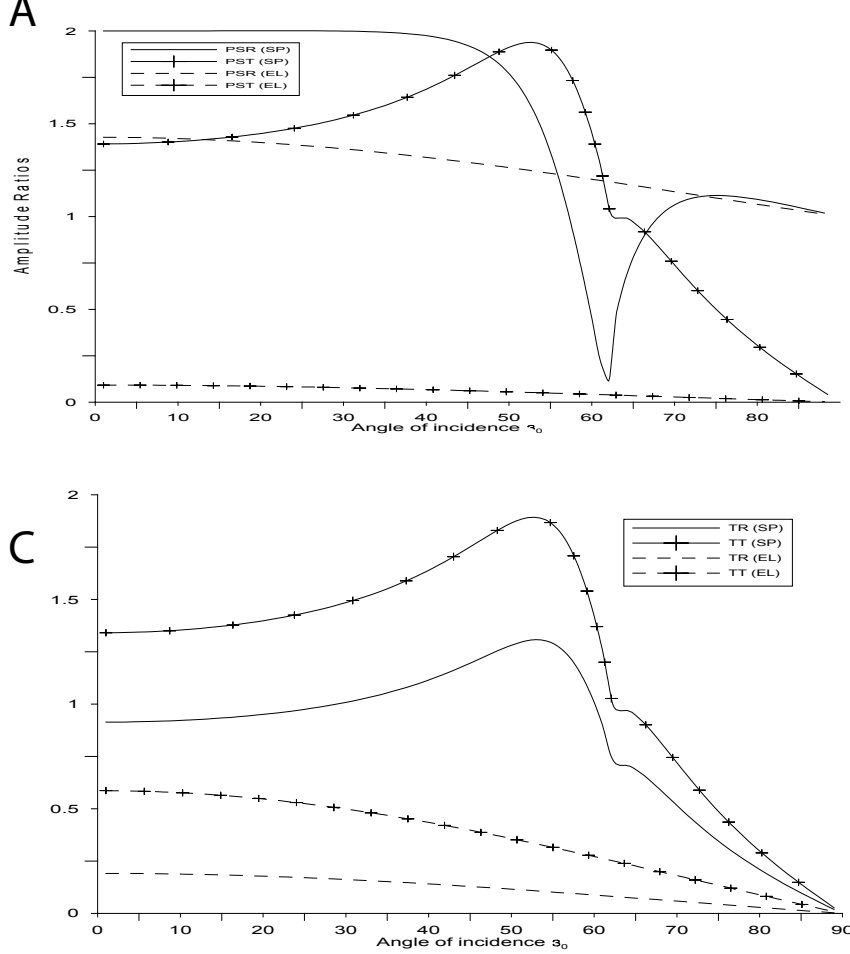

B
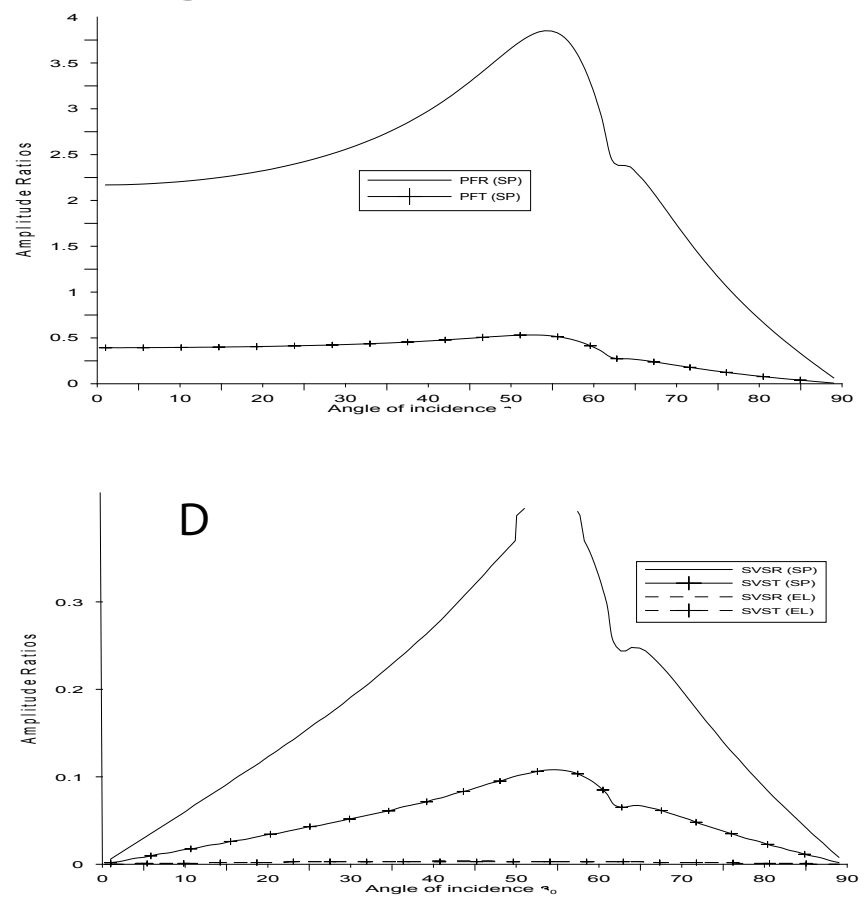

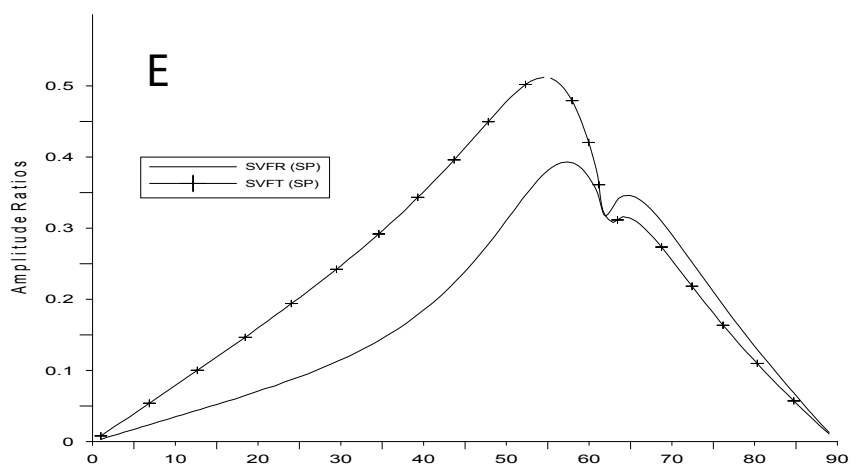

Figure 2 (a)-2(e): Represents the variation in amplitude ratios when PS wave is incident

$A^{*}=B_{04}, A_{01}=A_{02}=A_{03}=B_{05}=0$

$Y_{1}=a_{14}, \quad Y_{2}=a_{24}, \quad Y_{3}=-a_{34}, \quad Y_{4}=-a_{44}, Y_{5}=-a_{54}$

$Y_{6}=-a_{64}, \quad Y_{7}=a_{74}, \quad Y_{8}=a_{84}, Y_{9}=0, \quad Y_{10}=0$

(iv) For incident SVF -wave:

$$
\begin{aligned}
& A^{*}=B_{05}, A_{01}=A_{02}=A_{03}=B_{04}=0 \\
& Y_{1}=a_{15}, \quad Y_{2}=a_{25}, \quad Y_{3}=-a_{35}, \quad Y_{4}=-a_{45}, Y_{5}=-a_{55}, \\
& Y_{6}=-a_{65}, \quad Y_{7}=a_{75}, \quad Y_{8}=a_{85}, Y_{9}=0, \quad Y_{10}=0
\end{aligned}
$$$$
V_{0}=\left\{\begin{array}{cc}
V_{1} & \text { forincident } P S-\text { wave } \\
V_{2} & \text { forincident } P F-\text { wave } \\
V_{3} & \text { forincident } T \text {-wave } \\
V_{4} & \text { for incident } S V S-\text { wave } \\
V_{5} & \text { for incident } S V F-\text { wave }
\end{array}\right\}
$$

$$
\left|Z_{s}\right|=Z_{s} \quad s=1,2,3,4,5,6,7,8,9,10
$$

\section{Numerical Results and Discussion}

In order to illustrate theoretical results obtained in the preceding sections, we now present some numerical results. For numerical computation, the physical data is given below:

\footnotetext{
$\lambda=2.238 \times 10^{10} \mathrm{~N} / \mathrm{m}^{2}, \mu=2.992 \times 10^{10} \mathrm{~N} / \mathrm{m}^{2}$,

$\lambda_{v}=2.05 \times 10^{10} \mathrm{NSec} / \mathrm{m}^{2}, \mu_{v}=2.5 \times 10^{10} \mathrm{NSec} / \mathrm{m}^{2}$,

$\sigma^{f}=1.42 \times 10^{10} \mathrm{~N} / \mathrm{m}^{2}, \sigma^{f f}=1.75 \times 10^{10} \mathrm{~N} / \mathrm{m}^{2}$,

$\rho_{0}^{s}=2.65 \times 10^{3} \mathrm{NS} \mathrm{ec}^{2} / \mathrm{m}^{4}, \rho_{0}^{f}=1.92 \times 10^{3} \mathrm{NS} \mathrm{ec}^{2} / \mathrm{m}^{4}$

$\xi^{f f}=1.745 \times 10^{3} \mathrm{NSec} / \mathrm{m}^{4}, \gamma^{f}=0.25 \times 10^{6} \mathrm{~N} / \mathrm{m}^{2}{ }^{0} \mathrm{c}$,

$\alpha^{f}=1.152 \times 10^{6} \mathrm{~N} / \mathrm{m}^{20} \mathrm{c}, \alpha_{1}=0.03831 \times 10^{2} \mathrm{~N} / \mathrm{m}^{2}{ }^{0} \mathrm{c}^{2}$

$\zeta^{f}=0.35 \times 10^{6} \mathrm{~N} / \mathrm{m}^{2}, T_{0}=8.954^{0} c, K^{*}=0.386 \times 10^{2} \mathrm{~N} / \mathrm{sec}^{0} c$,

$\alpha_{0}=1.35 \times 10^{6} \mathrm{~N} / \mathrm{m}^{2}{ }^{0} \mathrm{c}, \varpi=0.05 \times 10^{2} \mathrm{~N} \mathrm{sec} / \mathrm{m}^{2}{ }^{0} \mathrm{c}^{2}$
} 

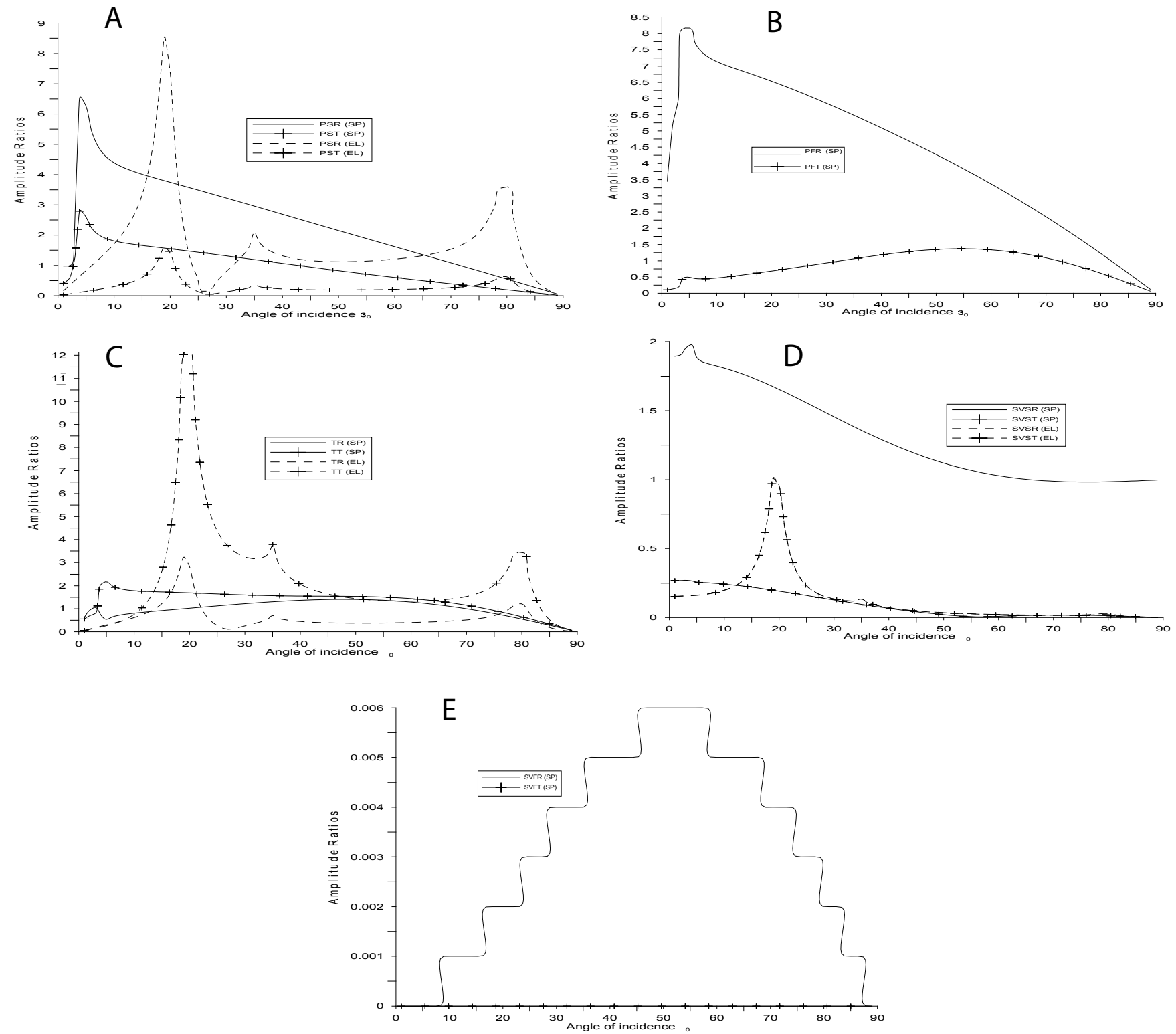

Figure 3(a)-3(e): Depicts the variation in values of amplitude ratio when SVS wave is incident

$\bar{\lambda}=0.91 \times 10^{10} \mathrm{~N} / \mathrm{m}^{2}, \bar{\mu}=1.11 \times 10^{10} \mathrm{~N} / \mathrm{m}^{2}, \bar{\lambda}=1.15 \times 10^{10} \mathrm{NSec} / \mathrm{m}^{2}$,

$\bar{\mu}_{v}=1.29 \times 10^{10} \mathrm{NSec} / \mathrm{m}^{2}, \bar{\sigma}^{f}=0.7 \times 10^{10} \mathrm{~N} / \mathrm{m}^{2}, \bar{\sigma}^{f f}=0.5 \times 10^{10} \mathrm{~N} / \mathrm{m}^{2}$,

$\bar{\rho}_{0}^{s}=1.25 \times 10^{3} \mathrm{NS} \mathrm{ec} / \mathrm{m}^{4}, \bar{\rho}_{0}^{f}=0.12 \times 10^{3} \mathrm{NS} \mathrm{ec}^{2} / \mathrm{m}^{4}$,

$\bar{\xi}^{f f}=0.1 \times 10^{3} \mathrm{NSec} / \mathrm{m}^{4}, \bar{\gamma}^{f}=0.15 \times 10^{6} \mathrm{~N} / \mathrm{m}^{2{ }^{0} \mathrm{c}}, \bar{\alpha}^{f}=0.152 \times 10^{6} \mathrm{~N} / \mathrm{m}^{20} \mathrm{c}$,

$\bar{\alpha}_{1}=0.3831 \times 10^{2} \mathrm{~N} / \mathrm{m}^{2}{ }^{0} \mathrm{c}^{2} \bar{\zeta}^{f}=0.45 \times 10^{6} \mathrm{~N} / \mathrm{m}^{2}, \bar{T}_{0}=1.24^{0} \mathrm{c}$,

$\bar{\alpha}_{0}=0.25 \times 10^{6} \mathrm{~N} / \mathrm{m}^{2}{ }^{0} \mathrm{c}, \overline{\bar{\sigma}}=0.02 \times 10^{2} \mathrm{~N} \mathrm{sec} / \mathrm{m}^{2}{ }^{0} \mathrm{c}^{2}$

The solid lines with centre symbols represent the swelling porous medium whereas small dashes lines represent the elastic medium (EL). Variations of amplitude ratios $Z_{s}$ with the angle of incidence $\theta_{0}$ for different waves are shown in Figure 2.

\section{Reflection and Transmission}

\section{PS wave}

Figure 2(a) depicts the variation in values of amplitude ratio $\left|Z_{1}\right|$ when PS wave is incident. From the figure it is noticed that values of amplitude ratios in SP medium are of oscillatory behavior whereas for EL medium it decrease with angle of incidence. In EL medium values of amplitude ratio for PST remains less than the values obtain for PSR in the whole range.

Figure 2(b) shows that values of amplitude ratio for PFR(SP) and $\mathrm{PFT}(\mathrm{SP})$ are of oscillatory behavior. The values of amplitude ratio for PFR increase in range , $1 \leq \theta_{0} \leq 50$ then starts decreasing whereas for PFT, increase in region, $1 \leq \theta_{0} \leq 55$ then decreases as $\theta_{0}$ increase further. For whole range the values of amplitude ratio obtain for transmitted part remains less than the values obtain for reflected part of $\mathrm{PF}$ wave.

From Figure 2(c) it is noticed that values of amplitude ratio obtain in SP medium are of oscillatory behavior whereas for EL medium the values decrease with angle of incidence. In SP medium the amplitude 
Citation: Kumar R, Taneja D, Kumar K (2012) Reflection and Transmission at the Boundary Surface of Thermoelastic Swelling Porous Media. J Appl Mech Eng 1:104. doi:10.4172/2168-9873.1000104

values for transmitted part of $\mathrm{T}$ wave remains greater than the reflected part of $\mathrm{T}$ wave whereas for EL medium, reverse behavior is noticed.

Figure 2(d) shows that values of amplitude ratio obtain in SP medium and EL medium are of oscillatory behavior. For EL medium, the values of amplitude ratio for reflected part of SVS wave remains slightly less than the values for transmitted part of SVS wave, whereas for SP medium, values of amplitude ratio for reflected part of SVS wave are more than the values obtained for transmitted part of SVS wave.

From Figure 2(e), we notice that for SP medium values of amplitude ratio for SVFR and SVFT are of oscillatory behavior. In range, $1 \leq \theta_{0} \leq 56$ values of amplitude ratio for SVFR are less than the values for SVFT, whereas for $\theta_{0} \geq 57$ reverse behavior is noticed. The values of amplitude ratio for SVFR(SP) and SVFT(SP) are magnified by $10^{5}$.

\section{SVS wave}

Figure 3(a) depicts the variation in values of amplitude ratio of reflected and transmitted part of PS wave for SP and EL medium. From the figure it is noticed that for EL medium the values of amplitude ratio obtained for reflected and transmitted part of PS wave oscillate in whole range, whereas for SP medium, initially oscillates then decreases. The transmitted part of PS wave remains less than the reflected part for the both the medium.

Figure 3(b) shows that the values of amplitude ratio for PFR increase in range $1 \leq \theta_{0} \leq 6$, then decrease whereas for PFT, oscillate in the whole region. The values of amplitude ratio obtained for transmitted part of $\mathrm{PF}$ wave remains less than the values obtain for reflected part in whole range.

From Figure 3(c), it is noticed that values of amplitude ratio for TR and TT oscillate in whole range. The values of amplitude ratio obtain for reflected part of $\mathrm{T}$ wave remains less than the transmitted part for both SP medium and EL medium.

Figure 3(d) shows that for SP medium, the values of amplitude ratio for SVSR initially oscillate then decrease with angle of incidence whereas for SVST it decrease in whole range. The values of amplitude ratios for SVST (SP) remain less than the values of SVSR (SP) in the whole range. In EL medium the values of amplitude ratio for SVSR remains less than the values obtained for SVST. The values of amplitude ratio for SVSR (EL) and SVST (EL) are of oscillatory behavior in whole range.

Figure 3(e) shows that the value of amplitude ratio for SVFR increase in range $1 \leq \theta_{0} \leq 55$, then decrease, whereas for SVFT increase in range $1 \leq \theta_{0} \leq 40$ the decreases. The values of amplitude ratios obtained for transmitted part of SVF wave remain less than the values obtained for reflected part of SVF wave in the whole region. The values of amplitude ratios of reflected and transmitted part of SVF wave are magnified by $10^{4}$.

\section{Conclusion}

From the above figures, we conclude that when PS wave is incident the values of amplitude ratios for SP medium are of oscillatory behavior whereas for EL medium the values decrease with angle of incidence. When SVS wave is incident the values of amplitude ratios are of oscillatory behavior in both SP and EL medium. The values of amplitude ratios for transmitted part of PS, SVS wave remains less than the values obtain for the reflected part of corresponding waves.

\section{References}

1. Eringen AC (1994) A Continuum theory of swelling porous elastic soils. Int $J$ Eng Sci 32: 1337-1349.
2. Biot MA (1956) Theory of propagation of elastic waves in a fluid saturated porous solid-I. Low frequency range. J Acoust Soc Am 28: 168-178.

3. Biot MA (1956) Theory of propagation of elastic waves in a fluid saturated porous solid-II. Higher frequency range. J Acoust Soc Am 28: 179-191.

4. Fillunger $P(1933)$ Der Auftrieb in Talsperren Osterr Wochenschrift fur den oftemtl. Bauelienst. I. Teil 532-552, III Teil: 567-570.

5. Bowen RM (1980) Incompressible Porous Media Models by use of the Theory of Mixtures. Int J Eng Sci 18: 1129-1148.

6. Boer R, Ehlers W (1990) The development of the concept of Effective Stress Acta Mechanica 83: 77-92.

7. De Boer R, Ehlers W (1990) Uplift, Friction and Capillarity: Three Fundamenta Effects for Liquid-saturated porous solid. Int J Solids Struct 26: 43-57.

8. Ehlers W (1993) Compressible, incompressible and hybrid two-phase models in porous theories. ASME; AMD 158: 25-38.

9. Angel YC, Achenbach JD (1985) Reflection and Transmission of elastic waves by a periodic array of cracks. J App Mech 52: 33-41.

10. Tomar SK (1995) Reflection and Refraction of longitudinal displacement wave at a liquid micropolar solid interface. Int J Eng Sci 33: 1507-1515.

11. Sinha SB, Elasibai KA (1997) Reflection and Refraction of thermoelastic wave at an interface of two semi-infinite media with two relaxation times. Journal of Thermal stresses 20: 129-145.

12. Singh B, Kumar R (1998) Reflection and Refraction of plane waves at an interface between micropolar elastic solid and viscoelastic solid. Int J Eng Sci 36: 119-135.

13. Singh B, Kumar R (2000) Reflection and Refraction of micropolar elastic waves at an interface between liquid-saturated porous solid and micropolar elastic solid. Proc Nat Acad Sci 70: 397-410.

14. Tomar S, Kumar R, Chopra A (2002) Reflection and Refraction of SH-Waves at corrugated interface between transversely isotropic and visco-elastic solid half space. Acta geophysica Polonica 50: 231-249.

15. Kumar R, Sarthi P (2006) Reflection and refraction of thermoelastic plane waves at an interface between two thermoelastic media without energy dissipation. Arch Mech 58: 155-185.

16. Chattopadhyay A, Gupta S, Sharma VK, Kumari P (2009) Reflection and Refraction of plane quasi- $P$ waves at a corrugated interface between distinct triclinic elastic half-spaces. Int J Solids Struct 46: 3241-3256.

17. Schoenberg M (1971) Transmission and reflection of plane waves at an elasticviscoelastic interface. Geophysical Journal of the Royal Astronomical Society 25: $35-47$ 